\title{
1 Viral Infection Skews Immunoglobulin Light Chain Repertoire Diversity
}

\section{John C. Schwartz ${ }^{1,2}$ and Michael P. Murtaugh ${ }^{1 \dagger}$}

$3{ }^{1}$ Department of Veterinary and Biomedical Sciences, University of Minnesota, 1971 Commonwealth

4 Avenue, St. Paul, MN 55108, USA.

$5 \quad{ }^{2}$ Current Address: The Pirbright Institute, Pirbright, Surrey GU24 0NF, UK

6

$7 \quad{ }^{\dagger}$ Corresponding author

$8 \quad$ Phone: $612-625-6735$

9 E-mail: murta001@umn.edu

\section{Acknowledgements}

13 We thank Juan Abrahante for assistance and advice in sequence data management and Gayathri Dileepan

14 for technical assistance. Funding was provided by the National Pork Board grant 10-139 (J.C.S. and

15 M.P.M.). J.C.S. was supported by the Molecular Virology Training Grant T32 AI83196 from the National

16 Institutes of Health, a Doctoral Dissertation Fellowship from the University of Minnesota, and is

17 currently supported by the United Kingdom Biotechnology and Biological Sciences Research Council

18 (BBSRC) project BBS/E/I/00001710, "Exploring the impact of genetic variation on the livestock immune

19 response to pathogens."

\section{Conflict of interest statement}

22 The authors declare no conflicts of interest.

\section{$24 \quad$ Keywords}

25 Sus scrofa; porcine; light chain; repertoire; antibody; allele; PRRSV 


\section{Highlights}

- $\quad \lambda$ and $\kappa$ light chain diversity is equivalent to heavy chain diversity

- High diversity is present despite limited gene segment usage

- PRRSV infection increases abundance of dominant $\lambda$ and $\kappa \mathrm{VJ}$ clones

- High levels of variation are present among animals

\section{Abstract}

Antibody responses are fundamentally important to effector and memory mechanisms of disease

resistance. Antibody repertoire diversity and its response to natural infection is poorly understood, yet is a prerequisite for molecular and structural elucidation of functionally protective immunity to viral infections. Using a swine model of mammalian viral infection, we observed marked changes following infection with the major porcine pathogen, porcine reproductive and respiratory syndrome virus (PRRSV). Deep sequencing of $>516,000$ light chain VJ mRNA genes showed that, similar to humans,

39 swine utilize both lambda and kappa loci equivalently. However, V and J gene usage were highly restricted; $\geq 99 \%$ of lambda light chains were $I G L V 3$ and $I G L V 8$ family members joined to $I G L J 2$ or $I G L J 3$, and $100 \%$ of kappa locus transcripts were $I G K V 1$ or $I G K V 2$ with only IGKJ2. Complementaritydetermining region (CDR) variation was limited. Nevertheless, total diversity richness estimates were 2.3

$43 \times 10^{5}$ for lambda and $1.5 \times 10^{5}$ for kappa, due in part to extensive germline variation in framework regions

44 and allelic variation. Infection by PRRSV reduced total richness due to expression of several highly

45 abundant clonal populations. Antibody light chain repertoires differed substantially among individuals,

46 thus illustrating extensive potential variation in immune response in outbred populations. These findings

47 demonstrate that individual variation in light chain repertoires may be an important component of variable

48 antibody responses to infection and vaccination, and that swine are a relevant model of human antibody

49 diversification in which the immune response capacity is critical to understanding individual variation in

50 immune protection against disease. 


\section{Introduction}

53 Outbred populations of humans and animals show extensive individual variation in the efficacy of

54 immune responses to natural infections and vaccinations, even though genetic and biochemical

55 mechanisms used to generate diversity within individual animals have an enormous capacity for

56 recognition of antigenic structures. For example, the BCG (Bacillus Calmette-Guérin) vaccine provides a

57 wide range of protection against tuberculosis in humans, ranging from nearly ineffective to nearly

58 completely effective $(1,2)$. Vaccination of pigs against foot and mouth disease virus (FMDV) is

59 characterized by significant animal-to-animal variation in anti-FMDV IgA (3); and post-vaccination anti-

60 FMDV antibody responses in cattle are significantly affected by genetic background (4). Furthermore in

61 swine there is substantial antibody response variation to vaccination against Actinobacillus

62 pleuropneumoniae and to infection with Mycoplasma hyorhinis $(5,6)$. While many factors may account

63 for individual variation in the immune response to an antigen, the size of the antibody repertoire and

64 variation in repertoire composition could be significant host-dependent factors.

66 Swine are susceptible to a range of bacterial and viral contagions similar or identical to those in humans.

67 As in other species, the porcine immunoglobulin genes are organized in three loci: a heavy chain locus on

68 chromosome 7 and kappa (IGK) and lambda (IGL) light chain loci on chromosomes 3 and 14,

69 respectively (7). We previously characterized the genomic organization of the kappa and lambda light

70 chain immunoglobulin loci $(8,9)$. Within the kappa locus, there are three functional IGKJ genes and the

71 first $14 I G K V$ genes have been identified, of which nine are putatively functional (8). The porcine lambda

72 locus possess two functional $I G L J$ genes and 12 putatively functional $I G L V$ genes belonging to three

73 families, of which the IGLV3 and IGLV8 families comprise the vast majority of known expression $(9,10)$.

74 We also previously identified substantial allelic variation among the germline $I G L V(11)$ and described

75 IGLV3-6 which is present in some animals as either a pseudogene or as a functional highly expressed

76 gene (12). 
78 To further analyze the diversity of the light chain repertoire and its response to disease challenges, we

79 sequenced and analyzed antibody light chain amplicon libraries obtained from healthy and virally-

80 infected pigs. The analyses confirmed limited combinatorial diversity, yet extensive complementarity-

81 determining region (CDR) and framework variation, likely arising from somatic hypermutation. Marked

82 changes in abundance and repertoire diversity were observed following porcine reproductive and

83 respiratory syndrome virus (PRRSV) infection that were indicative of clonally amplified and potentially

84 PRRSV-specific B cells. Our observations also suggest that the utilization (or lack thereof) of IGLV3-6

85 may skew the antibody repertoire toward expressing other genes at higher levels.

87 Materials and Methods

88 Samples

89 The animal studies were reviewed and approved by the University of Minnesota Institutional Animal Care

90 and Use Committee. Lymphoid tissues (spleen, palatine tonsil, inguinal lymph node, and bronchial lymph

91 node) from five genetically-similar, commercially-sourced piglets were obtained from a previous study

92 (13). Piglets were challenged with virulent PRRSV strain JA142 ( $n=3)$ or not ( $n=2)$ at 3-4 weeks of age

93 and tissues were harvested 63 days after infection and stored in RNAlater (Ambion/Thermo-Fisher).

94 Tissues were homogenized and total RNA was extracted using the RNeasy Mini Kit (Qiagen) and reverse

95 transcribed using the QuantiTect Reverse Transcription Kit (Qiagen).

97 Library generation and sequencing

98 Primers were designed in Primer3 set to default parameters (14). IGKV and IGLV gene family-specific

99 forward primers were designed using the conserved leader region sequences from annotations of the

100 porcine kappa and lambda loci. In order to detect possible functional alleles of previously annotated

101 pseudogenes, forward primers were designed such that all known kappa and lambda $\mathrm{V}$ genes could be

102 amplified from cDNA regardless of functionality. Hence, the leader region for IGLV8-21, a pseudogene, 
was distinct enough to warrant a separate forward primer (Table 1). Reverse primers were designed to be

104 specific for $I G K C$ and the three nearly identical $I G L C$ genes $(8,9)$.

The Roche 454 Titanium fusion adapter A (5'-CGTATCGCCTCCCTCGCGCCATCAG) was added to

107 the 5' end of each forward primer and fusion adapter B (5'-CTATGCGCCTTGCCAGCCCGCTCAG) was

108 added to the $5^{\prime}$ end of each reverse primer. Ten base pair molecular ID tags were added to the forward

109 primer between the fusion adapter and the template-specific sequence to identify animal source. PCR

110 reactions were performed separately by tissue and products were pooled according to pig, quantified, and

111 re-pooled in equimolar amounts so that the final library contained approximately the same number of

112 amplicons from each animal. Pooled amplicons were gel-purified using the QIAquick gel extraction kit

113 (Qiagen). To remove trace amounts of agarose, the amplicons were purified again using the QIAquick

114 PCR purification kit (Qiagen). Roche Titanium 454 pyrosequencing was performed at the W. M. Keck

115 Center for Comparative and Functional Genomics, University of Illinois at Urbana-Champaign.

117 Sequence analysis

118 Pyrosequencing reads were deposited in the National Center for Biological Information (NCBI) sequence 119 read archive (SRA) database under accession number SRP026119 (BioProject: PRJNA206406). Short

120 reads $(<350 \mathrm{bp})$, reads with a missing or aberrant molecular ID tag, and reads containing premature stop 121 codons were excluded from further analyses. IGKV and $I G L V$ gene usage was determined using BLAST

122 (15). Reads were translated into putative amino acid sequences using EMBOSS within the Galaxy

123 framework $(16,17)$. For diversity analysis, amino acid sequences were first clustered using CD-HIT at an

124 identity threshold of $100 \%$ (18) and these clusters were used to generate abundance curves and richness

125 estimates. CD-HIT and Chaol were used to calculate lower-bound estimates of repertoire richness (19-

126 21). The clustered results were used to generate abundance curves within Microsoft Excel. Total number

127 of sequence clusters and the numbers of singletons and doubletons generated from CD-HIT output were

128 used to calculate Chao1 lower-bound estimates of repertoire richness (19-21). 


\section{Results}

\section{Sequencing results}

132 A total of 516,097 pyrosequencing reads were obtained with a mean length of approximately $510 \mathrm{bp}$ and a

133 median length of $521 \mathrm{bp}$. Reads shorter than $350 \mathrm{bp}$ which did not span the entire variable region were

134 removed from further analyses (79,172 reads). An additional 11,739 reads with aberrant molecular ID

135 tags and 53,046 reads containing premature stop codons and/or ambiguous amino acids were also

136 removed from further analyses. Thus, a total of 372,140 full-length, in-frame reads were analyzed for V

137 and $\mathrm{J}$ gene usage. The percentages of reads from individual pigs were approximately equal (pig 1, 19.0\%;

138 pig 2, 16.9\%; pig 3, 20.3\%; pig 4, 20.0\%; pig 5, 23.8\%). Furthermore, the number of reads obtained from

139 kappa and lambda-containing transcripts were similar (42.9\% vs. 57.1\%, respectively).

IGK expression

142 A total of 159,721 reads corresponding to IGK and covering the entire variable region were obtained.

143101,714 reads (64\%) matched most closely to genes from the IGKVI family and 58,007 matched most

144 closely to the $I G K V 2$ family (Fig. 1A). IGKJ2 accounted for the vast majority of J gene usage (Fig. 1A).

145 In contrast, IGKJ3 and IGKJ5 were not expressed and both $I G K J 1$ and $I G K J 4$ were expressed at very low

146 levels (approximately $1 \%$ of all $I G K J$ genes). There was no discernable association between $I G K J$ and

$147 I G K V$ gene usage. Compared to the annotated germline sequences for $I G K V$, the median number of

148 predicted amino acid mismatches varied from 3 for $I G K V 2-6$ to 10 for $I G K V 1-11$ (Fig. 1E). No major

149 differences in $I G K V$ gene usage were noted between individual pigs. Of the nine putatively functional

$150 I G K V$ genes, two (IGKVI-7 and IGKVI-14) were not expressed in any of the five pigs.

152 IGL expression

153 A total of 212,419 full-length lambda variable region reads were obtained. IGLV gene BLAST results

154 were dominated by $I G L V 8$ family members (67.6\%), followed by $I G L V 3$ family members (32.4\%), and 
155 low expression (0.03\%) of IGLV5-14 also was observed (Fig. 1B). IGLJ gene usage was divided between

$156 I G L J 2$ (59\% of total reads) and IGLJ3 (41\% of total reads), and neither IGLJI nor IGLJ4 were expressed

157 (Fig. 1B). There was no association between specific $I G L V$ and $I G L J$ genes, as was observed for IGK.

158 One apparently functional gene, $I G L V 2-6$, showed no expression in any of the five animals, even though a

159 conserved forward primer specific for the IGLV2 leader region was used for PCR amplification (Table 1),

160 and post-PCR purification of a gel slice in the expected size range was included in the amplicon pool that

161 was sequenced. The PCR amplifications for the pseudogene IGLV8-21 typically yielded a faint band of

162 appropriate size. However, our sequence analysis failed to identify transcripts from this gene, suggesting

163 that these products were non-specifically amplified transcripts from other members of the IGLV 8 family.

165 Of the IGLV3 genes, IGLV3-3 and IGLV3-6 are the most highly expressed, yet this expression varies

166 substantially between individuals. Pig 1 is known to be homozygous for a deletion of IGLV3-6 and pig 2

167 likely possesses a different allele (12). In these animals, IGLV3-6 transcripts were therefore either absent

168 entirely or expressed at a relatively low level, respectively, compared to the other individuals. In contrast,

169 IGLV3-3 expression was higher in pigs 1 and 2 compared to the others (Fig. 1D). The usage of IGLV3-6

170 was variable between the five pigs (12). Interestingly, in pig 1, which was previously found to completely

171 lack this gene, IGLV3-3 was almost exclusively the only IGLV3 family member expressed (Fig. 1D). In

172 the other animals found to possess a functional copy, however, IGLV3-6 was one of the most highly

173 expressed genes in the light chain repertoire (Fig. 1D).

175 Light chain CDR3 length variation

176 Although a hallmark of heavy chains, CDR3 length variability is typically low in light chains, due to the

177 lack of terminal deoxynucleotidyl transferase (TdT) activity during light chain rearrangement (22).

178 However, we did observe some variation among the putatively functional light chain transcripts.

179 Approximately $12.5 \%$ of IGK CDR3s differed in length from the germline encoded 9 amino acids, with a 
range of 4 to 16 amino acids. In contrast, 33.5\% of IGL CDR3s differed from the canonical 10 or 11

181 amino acids, ranging from 4 to 23 amino acids in length (Fig. 2).

\section{Diversity and richness of the IGK and IGL repertoires}

184 Log-log abundance curves indicate a power-law distribution in which the vast majority of light chain

185 sequences are exceptionally rare and a small number are very common (Fig. 3). The most abundant clones

186 represented $>1 \%$ of the total repertoire, while more than $50 \%$ of all sequences were singletons (IGK:

$18751.2 \pm 5.85 \%$; IGL: $55.4 \pm 6.96 \%$ (mean \pm 1 standard deviation) $)$. Chao1 repertoire richness was estimated

188 using deduced amino acid sequences for the full length V-J region from each animal. Lower bound

189 richness estimates of $1.6 \times 10^{5}$ to $2.8 \times 10^{5}$ molecules per pig were obtained for the IGL repertoire and

190 estimates of $1.1 \times 10^{5}$ to $1.7 \times 10^{5}$ molecules per pig were obtained for the IGK repertoire (Fig. 4). Their

191 sum approximates the estimated richness of the porcine heavy chain VDJ $\left(\sim 2.7 \times 10^{5}\right.$; Schwartz and

192 Murtaugh, unpublished data).

194 The presence of dramatic allelic variation in some V genes, particularly for IGLV3-6, suggests that 195 framework variation in the $I G L V$ and $I G K V$ family members might contribute to light chain repertoire 196 diversity (12). Phylogenetic analysis showed high levels of nucleotide sequence variation within and 197 between expressed light chain family members, varying by up to $11 \%$ and $15 \%$ pairwise differences 198 within $I G K V I$ and $I G K V 2$, respectively, and $8 \%$ and $28 \%$ in $I G L V 8$ and $I G L V 3$, respectively, while the 199 highest pairwise similarity between family members varied from $43 \%$ (IGKVI versus IGLV5) and $62 \%$

200 (IGLV3 versus IGLV8) (Fig. 8). The framework region of IGLV3-3 is also distinct from the other IGLV3

201 family members (Fig. 5). Within each group, variation is present in framework and CDR regions, with 202 only the CDR3 region showing extensive variation, in part because of CDR3 length variability. 
PRRSV infection caused a shift in light chain abundance distributions toward fewer, more abundant light chain sequences. Individual clonal populations were identified that accounted for one to three percent of the entire repertoires of individual pigs (Fig. 3). Individual variation in the abundance of specific clones is striking. The most abundant $I G L V-I G L J$ clone in this study, clone A in pig 1, was not present in uninfected pigs 3 and 4 and also was rare in PRRSV-infected pig 3 (Supplemental Table 1; Supplemental Table 2). The five most abundant clones in PRRSV-infected pig 1 were also present in infected pigs 2 and 3, but the five most abundant clones in PRRSV-infected pig 3 were absent or rare in the other two

212 infected pigs. The five most abundant clones in pig 2 show somatic changes from the germline sequence,

213 but 5 of the 10 most abundant clones in pigs 1 and 3 are unchanged from germline, the same ratio as in

214 the dominant clones of the uninfected animals (Supplemental Table 1). Several highly abundant clones

215 (e.g. clones B, E, L, M, and R) are present in multiple animals, including both PRRSV-infected and

216 uninfected pigs (Supplemental Table 1). Amino acid sequence variation in abundant lambda clones was

217 due primarily to mutations in both framework and CDR regions. The CDR1 region showed 4 sequence

218 motifs with lengths of 5, $6(\mathrm{n}=2)$ and 9 amino acids, while CDR2 had no length variation and CDR3

219 length was $8 \pm 1$ amino acids with one exception of 11 aa (Supplemental Table 2).

221 Unlike the IGL, abundant kappa clones in every case were mutated from the germline sequence, with an 222 average of 7.4 amino acid differences in IGKV (Supplemental Table 3), compared to an average of 1.6 in 223 lambda clones. These differences are nearly entirely mutational, as there were no length differences in 224 CDR2 or CDR3, and only 3 length classes (6, 10, and 11 aa) in CDR1 (Supplemental Table 4). Kappa 225 clones A and E were dominant in both PRRSV infected and uninfected pigs (Supplemental Tables 3). By 226 contrast, pig 3 possessed clones I, J, and K which were not present in any other pig. Except for two

$227 I G K V 2-8$ clones in pig 2, all abundant clones were derived from either IGKV1-11 or IGKV2-10

228 (Supplemental Table 3). 
231 Unraveling of the mechanisms of antibody diversification in previous decades revealed combinatorial and

232 mutational machinery able to produce a nearly limitless array of antigen-binding variability.

233 Paradoxically, exposure of animal populations to an antigen consistently results in a wide range of

234 response intensities. Therefore, the functional antibody repertoire of animals must be limited and variable

235 among individual members of the population. Characterization of antibody repertoires and sources of

236 individual variation in repertoire diversity in the absence and presence of antigenic challenge is essential

237 to understand variation in antibody responses to infection and vaccination. Here we show that pigs, which

238 are susceptible to a wide variety of microbial infectious diseases, use both IGL and IGK loci to produce

239 repertoires in excess of 100,000 lambda and kappa light chains, even though V gene usage is limited and

240 CDR complexity is low.

242 The IGK repertoire in the present study is dominated by the expression of only two genes, IGKV2-10 and

243 IGKV1-11, of which the latter is more highly expressed than the former. This result contrasts previous

244 reports suggesting that $I G K V 2$ family members are more abundantly expressed, at least among pre-

245 immune piglets $(8,23)$. Two putatively functional $\mathrm{V}$ genes, IGKVI-7 and IGKVI-14, lack a canonical

246 octamer (ATCTGCAT) and both possess a non-canonical 5' splice site in their intron (GT -> GG). As

247 shown in Figure 2, there is a notably high level of sequence divergence in IGK transcripts from the

248 germline annotation of the 14 most C-proximal IGKV genes in the locus (9). Since a high degree of

249 heterozygosity was present, it is possible that individual diversity may be due to allelic variation, as has

250 been shown in $I G L V$ genes $(11,12)$. Allelic variation also has been shown in humans to significantly

251 affect repertoire diversity $(24,25)$. However, whereas previous studies focused on heavy chain repertoire

252 diversity and variation, we show here that light chain variation also makes a substantial contribution to

253 overall antibody diversity using mechanisms that do not involve large CDR3 length variation and amino

254 acid sequence replacement. Thus, generation of light chain repertoire diversity lies at the opposite end of

255 the spectrum from bovine heavy chain diversity generation characterized by unusually long and complex

256 CDR3 regions (26). 
258 Diversity estimates as well as the raw sequencing results indicate that lambda expression accounts for 55

259 to $60 \%$ of total light chains, and consists overwhelmingly of IGLV8 family members, followed by IGLV3

260 family members. These results are consistent with previous reports of the pig lambda repertoire $(10,27)$,

261 which also suggested that there are age-dependent changes in IGLV usage. Although putatively

262 functional, IGLV2-6 was not expressed in any of the five animals studied. Our previous analysis indicated

263 that this gene lacks a conserved octamer in the promoter region (ATTTGCAT $->$ ATTTGTAT) and a

264 conserved heptamer in the recombination signal sequence (CACAGTG -> TACAGTG), which together

265 appear to account for its lack of functional expression (9). Both $I G K V$ and $I G L V$ showed limited CDR

266 diversity but substantial framework variation that is most striking in the IGKV3 family. The broad

267 distribution of amino acid variation, encoded in the DNA, across the majority of the light chain VJ

268 regions, creates extensive diversity and, presumably, in the structural stability of the antibody molecule. It

269 also increases the likelihood that allelic variation will contribute substantially to variation in the antibody

270 responses of individual animals to antigenic challenge.

271

272 Viral infection skewed light chain responses to more highly abundant clones which is suggestive of

273 antigen-driven clonal expansion. It was previously argued that PRRSV induces a pronounced nonspecific

274 B-cell activation and hypergammaglobulinemia that also could skew antibody responses to abundant,

275 antigen-independent clones due to its persistence in the host. However, it is apparent now that PRRSV

276 infections are resolved completely, and that evidence is lacking for hypergammaglobulinemia in

277 conventional swine infected or vaccinated with PRRSV and reared under a variety of conditions (28). The

278 specificity of highly expressed light and heavy chains is resolvable due to the ability to clone and express

279 recombinant antibodies. Therefore, we expect that the antigen specificity of highly abundant clones will

280 be determined in later studies. 
282 Interestingly, pig 1 showed a marked reduction in total estimated richness in the lambda repertoire

283 compared to the other four individuals. The reduction appears to be due to genetic constraints in the C-

284 proximal IGLV3 gene cluster. In particular, the sequences obtained from this animal indicated a high

285 degree of IGL homozygosity. Within the C-proximal IGLV cluster, only IGLV3-3 was expressed at high

286 levels, while the other IGLV3 genes were nearly unused. In addition, IGLV3-6 expression was absent. As

287 the most abundantly expressed antibody sequence from this animal was of IGLV3-3 origin, it is possible

288 that the reduction in richness was due to genetic constraints and not PRRSV infection. Taken together, the

289 findings show that the light chain repertoire is equivalent in size and complexity to the heavy chain

290 repertoire, in the range of $10^{5}$ to $10^{6}$ unique light chains per individual, with fully half of the sequences

291 present as singletons. These richness estimates closely resemble the reported richness of the human light

$292\left(1.6 \times 10^{5}\right)$ and heavy $\left(2.2 \times 10^{5}\right)$ chain repertoires which were estimated using a capture-recapture

293 rarefaction method (38).

295 Analyses of the human light chain repertoire has revealed significant differences between individuals in

296 their CDR3 sequences and V gene usage $(29,30)$. Indeed, in a study consisting of four humans,

297 approximately 60 percent of light chain rearrangements were present in more than one individual (30).

298 Furthermore, approximately 20 percent of light chain CDR3s were shared by more than one individual in

299 a study consisting of five humans (29). Our data also show substantial individual variation in the

300 molecular distribution of light chains in pigs; the most dominantly expressed sequences in some animals

301 are not present in others. These features are also characteristic of antibody heavy chains in other species

302 such as zebrafish $(31,32)$. However, since zebrafish have only $\sim 300,000$ B-cells and a heavy chain

303 richness of 1200-3700 unique antibodies per individual $(31,32)$, the actual diversity of antibodies

304 produced in a population will be distributed stochastically among individuals such that broad variation in

305 ability to respond to antigens will be a feature of the population more than the individual. The spleen of

306 swine and humans, alone, has a mass more than 100 times larger than a zebrafish $(\sim 150 \mathrm{~g}$ versus $<1 \mathrm{~g}$,

307 (33-36)), and with many additional lymphoid tissues, the total B-cell compartment of humans and large 
308 animals is orders of magnitude larger than a zebrafish or mouse, whose spleen weighs about $100 \mathrm{mg}$ (37).

309 Therefore, swine are a relevant model of human antibody diversification in which the immune response

310 capacity of individuals is critical to understanding variation in the effectiveness of immunity to

311 pathogens. 


\section{References}

314 1. Colditz, G. A., T. F. Brewer, C. S. Berkey, M. E. Wilson, E. Burdick, H. V. Fineberg, and F.

315 Mosteller. 1994. Efficacy of BCG vaccine in the prevention of tuberculosis. Meta-analysis of the 316 published literature. J Am Med Assoc 271: 698-702.

317 2. Zodpey, S. P. 2004. The BCG controversy: a reappraisal of the protective effect against

318 tuberculosis and leprosy. Indian journal of public health 48: 70-77.

319 3. Francis, M. J., and L. Black. 1986. Humoral response of pregnant sows to foot and mouth disease vaccination. The Journal of hygiene 96: 501-511.

321 4. Di Giacomo, S., B. P. Brito, A. M. Perez, D. Bucafusco, J. Pega, L. Rodríguez, M. V. Borca, and M. Pérez-Filgueira. 2015. Heterogeneity in the antibody response to foot-and-mouth disease primovaccinated calves. Transbound Emerg Dis 62: 280-287.

324 5. Magnusson, U., J. Bosse, B. A. Mallard, S. Rosendal, and B. N. Wilkie. 1997. Antibody response to Actinobacillus pleuropneumoniae antigens after vaccination of pigs bred for high and low immune response. Vaccine 15: 997-1000.

327 6. Magnusson, U., B. Wilkie, B. Mallard, S. Rosendal, and B. Kennedy. 1998. Mycoplasma hyorhinis infection of pigs selectively bred for high and low immune response. Vet Immunol Immunopathol 61: 83-96.

7. Yerle, M., Y. Lahbib-Mansais, P. Pinton, A. Robic, A. Goureau, D. Milan, and J. Gellin. 1997. The

332 8. Schwartz, J. C., M. P. Lefranc, and M. P. Murtaugh. 2012. Evolution of the porcine (Sus scrofa domestica) immunoglobulin kappa locus through germline gene conversion. Immunogenetics 64:

335 9. Schwartz, J. C., M. P. Lefranc, and M. P. Murtaugh. 2012. Organization, complexity and allelic 
10. Wertz, N., J. Vazquez, K. Wells, J. Sun, and J. E. Butler. 2013. Antibody repertoire development in

11. Guo, X., J. C. Schwartz, and M. P. Murtaugh. 2016. Genomic variation in the porcine immunoglobulin lambda variable region. Immunogenetics 68: 285-293.

12. Schwartz, J. C., and M. P. Murtaugh. 2014. Characterization of a polymorphic IGLV gene in pigs (Sus scrofa). Immunogenetics 66: 507-511.

13. Klinge, K. L., E. M. Vaughn, M. B. Roof, E. M. Bautista, and M. P. Murtaugh. 2009. Agedependent resistance to Porcine reproductive and respiratory syndrome virus replication in swine. Virology journal 6: 177.

14. Rozen, S., and H. Skaletsky. 2000. Primer3 on the WWW for general users and for biologist programmers. Methods in molecular biology (Clifton, N.J.) 132: 365-386.

15. Altschul, S. F., W. Gish, W. Miller, E. W. Myers, and D. J. Lipman. 1990. Basic local alignment search tool. J Mol Biol 215: 403-410.

16. Rice, P., I. Longden, and A. Bleasby. 2000. EMBOSS: the European Molecular Biology Open Software Suite. Trends Genet 16: 276-277.

17. Blankenberg, D., J. Taylor, I. Schenck, J. He, Y. Zhang, M. Ghent, N. Veeraraghavan, I. Albert, W. Miller, K. D. Makova, R. C. Hardison, and A. Nekrutenko. 2007. A framework for collaborative analysis of ENCODE data: making large-scale analyses biologist-friendly. Genome Res 17: 960964.

18. Li, W., and A. Godzik. 2006. Cd-hit: a fast program for clustering and comparing large sets of protein or nucleotide sequences. Bioinformatics 22: 1658-1659.

19. Colwell, R. K., and J. A. Coddington. 1994. Estimating terrestrial biodiversity through extrapolation. Philos Trans R Soc Lond B Biol Sci 345: 101-118. 11: $265-270$. 
21. Chao, A., and S.-M. Lee. 1992. Estimating the number of classes via sample coverage. Journal of the American Statistical Association 87: 210-217.

22. Sun, X., N. Wertz, K. Lager, M. Sinkora, K. Stepanova, G. Tobin, and J. E. Butler. 2012. Antibody

23. Butler, J. E., N. Wertz, J. Sun, H. Wang, P. Chardon, F. Piumi, and K. Wells. 2004. Antibody

24. Boyd, S. D., B. A. Gaeta, K. J. Jackson, A. Z. Fire, E. L. Marshall, J. D. Merker, J. M. Maniar, L.

Egholm, D. B. Miklos, J. L. Zehnder, and A. M. Collins. 2010. Individual variation in the germline Ig gene repertoire inferred from variable region gene rearrangements. J Immunol 184: 6986-6992. chain V region gene loci by analysis of VDJ gene rearrangements. J Immunol 188: 1333-1340.

26. Wang, F., D. C. Ekiert, I. Ahmad, W. Yu, Y. Zhang, O. Bazirgan, A. Torkamani, T. Raudsepp, W. Mwangi, M. F. Criscitiello, I. A. Wilson, P. G. Schultz, and V. V. Smider. 2013. Reshaping antibody diversity. Cell 153: 1379-1393.

27. Guo, N., M. Su, Z. Xie, K. Wang, H. Yuan, M. Li, J. Li, M. Liu, J. Bai, J. Liu, H. Ouyang, D. Pang, and H. Jiao. 2018. Characterization and comparative analysis of immunoglobulin lambda chain diversity in a neonatal porcine model. Veterinary Immunology and Immunopathology 195: 84-91.

28. Murtaugh, M. P., and M. Genzow. 2011. Immunological solutions for treatment and prevention of porcine reproductive and respiratory syndrome (PRRS). Vaccine 29: 8192-8204.

29. Hoi, K. H., and G. C. Ippolito. 2013. Intrinsic bias and public rearrangements in the human immunoglobulin Vlambda light chain repertoire. Genes and immunity 14: 271-276. 
30. Jackson, K. J., Y. Wang, B. A. Gaeta, W. Pomat, P. Siba, J. Rimmer, W. A. Sewell, and A. M. Collins. 2012. Divergent human populations show extensive shared IGK rearrangements in peripheral blood B cells. Immunogenetics 64: 3-14.

31. Jiang, N., J. A. Weinstein, L. Penland, R. A. White, D. S. Fisher, and S. R. Quake. 2011. Determinism and stochasticity during maturation of the zebrafish antibody repertoire. Proc Natl Acad Sci U S A 108: 5348-5353.

32. Weinstein, J. A., N. Jiang, R. A. White, D. S. Fisher, and S. R. Quake. 2009. High-throughput sequencing of the zebrafish antibody repertoire. Science 324: 807-810.

33. Barrionuevo, W. R., and W. W. Burggren. 1999. O2 consumption and heart rate in developing zebrafish (Danio rerio): influence of temperature and ambient O2. Am J Physiol 276: R505-513.

34. Boisen, A. M., J. Amstrup, I. Novak, and M. Grosell. 2003. Sodium and chloride transport in soft water and hard water acclimated zebrafish (Danio rerio). Biochim Biophys Acta 1618: 207-218.

35. Walsh, R. M., B. Chand, J. Brodsky, and B. T. Heniford. 2003. Determination of intact splenic weight based on morcellated weight. Surg Endosc 17: 1266-1268.

36. Sprogøe-Jakobsen, S., and U. Sprogøe-Jakobsen. 1997. The weight of the normal spleen. Forensic Sci Int 88: 215-223.

37. Gibson, J. N., L. C. Jellen, E. L. Unger, G. Morahan, M. Mehta, C. J. Earley, R. P. Allen, L. Lu, and B. C. Jones. 2011. Genetic analysis of iron-deficiency effects on the mouse spleen. Mamm Genome 22: 556-562.

38. Lefranc, M. P., C. Pommié, M. Ruiz, V. Giudicelli, E. Foulquier, L. Truong, V. Thouvenin-Contet, and G. Lefranc. 2003. IMGT unique numbering for immunoglobulin and T cell receptor variable domains and Ig superfamily V-like domains. Dev Comp Immunol 27: 55-77. 
bioRxiv preprint doi: https://doi.org/10.1101/398529; this version posted August 23, 2018. The copyright holder for this preprint (which was not certified by peer review) is the author/funder, who has granted bioRxiv a license to display the preprint in perpetuity. It is made available under aCC-BY-NC-ND 4.0 International license.

Table 1. Primers used for amplification of porcine light chain cDNA VJ rearrangements.

\begin{tabular}{ll}
\hline Target & Template-specific sequence \\
\hline IGKV1 (F)* & 5'-GCCTCYTGCTGCTCTGG \\
IGKV2 (F) & 5'-TTCCCTGCTCAGCTCCTG \\
IGLV2 (F) & 5'-CCTTGTCACCCTCCTCACTC \\
IGLV3 (F) & 5'-CTGGAYCCCTCTCCTGCTC \\
IGLV5 (F) & 5'-GGACTCCTCTGCTGATCGTG \\
IGLV8 (F) & 5'-CCTGGACGGTGCTTCTGAT \\
IGLV8-21 (F) & 5'-CTTCTGCTTTGCTCCTCGTT \\
IGKC (R) & 5'-GACCACCCCATCCACTTTC \\
IGLC (R) & 5'-CCTTCCAGGTCACCGTCA \\
\hline
\end{tabular}


bioRxiv preprint doi: https://doi.org/10.1101/398529; this version posted August 23 2018. The copyright holder for this preprint (which was not certified by peer review) is the author/funder, who has granted bioRxiv a license to display the preprint in perpetuity. It is made available under aCC-BY-NC-ND 4.0 International license.

413 Figure 1. Light chain gene usage. $I G K V-I G K J(\mathrm{~A})$ and $I G L V-I G L J(\mathrm{~B})$ combinatorial usage. $I G K V(\mathrm{C})$

414 and $I G L V$ (D) gene usage in individual pigs. Deviation in amino acid sequence from the germline

415 reference sequence for $I G K V(\mathrm{E})$ and $I G L V(\mathrm{~F})$.

416

A

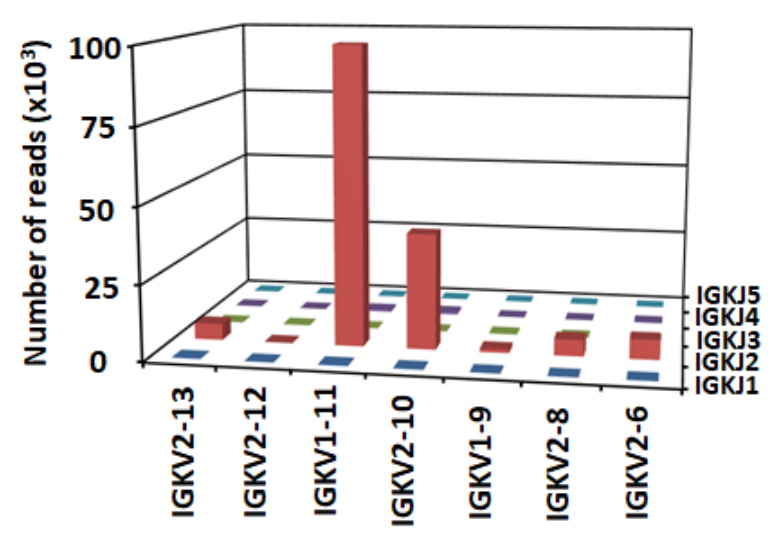

C

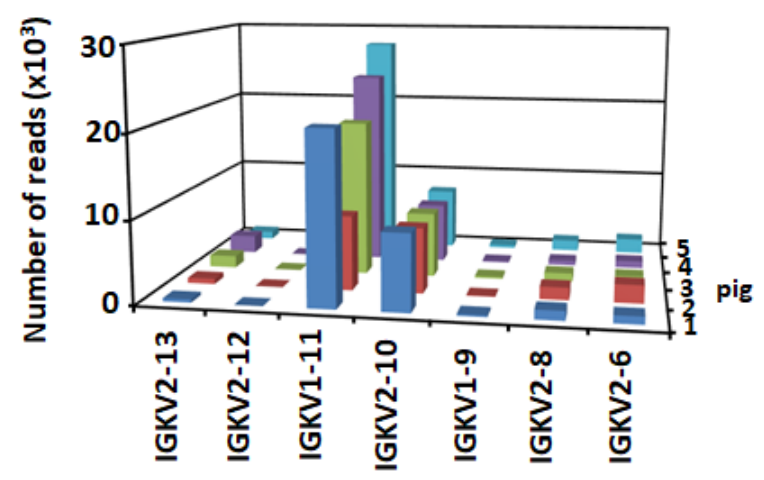

$\mathrm{E}$

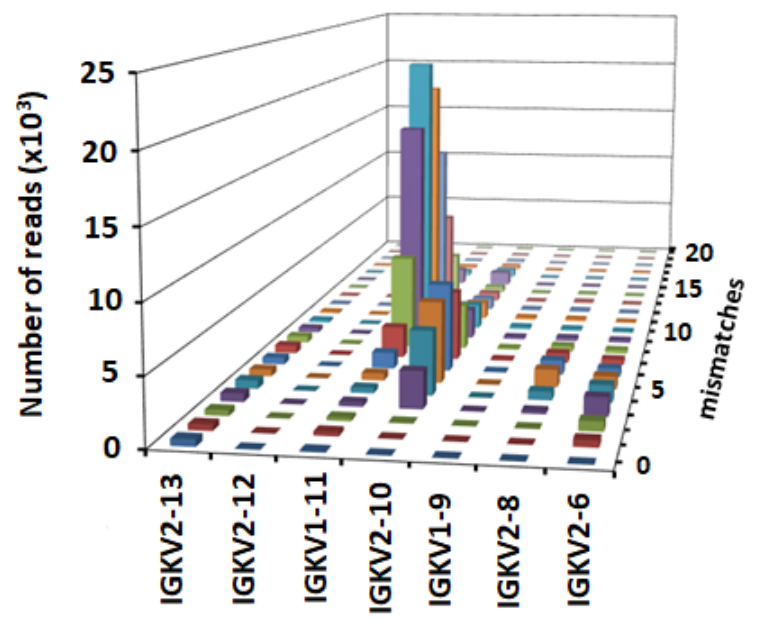

B



D

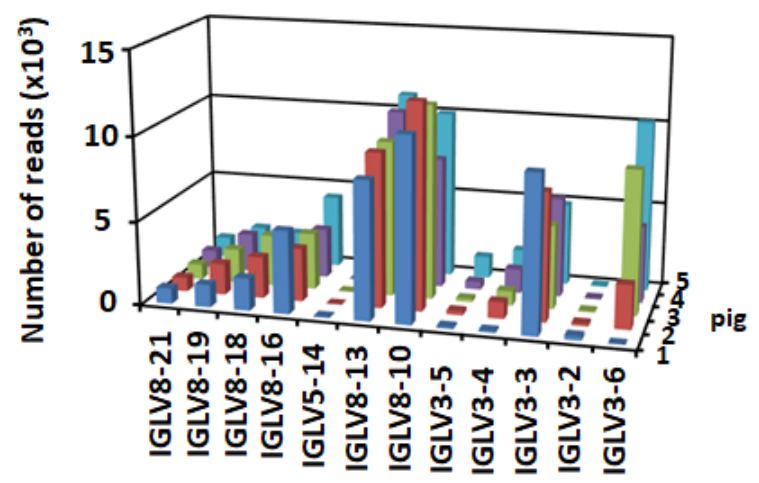

F




418 Figure 2. Length distributions of pig light chain CDR3. (A) Distribution of IGL CDR3 from 187,810

419 reads. (B) Distribution of IGK CDR3 from 147,189 reads.

A

424

425

426

427

428

429

430

431

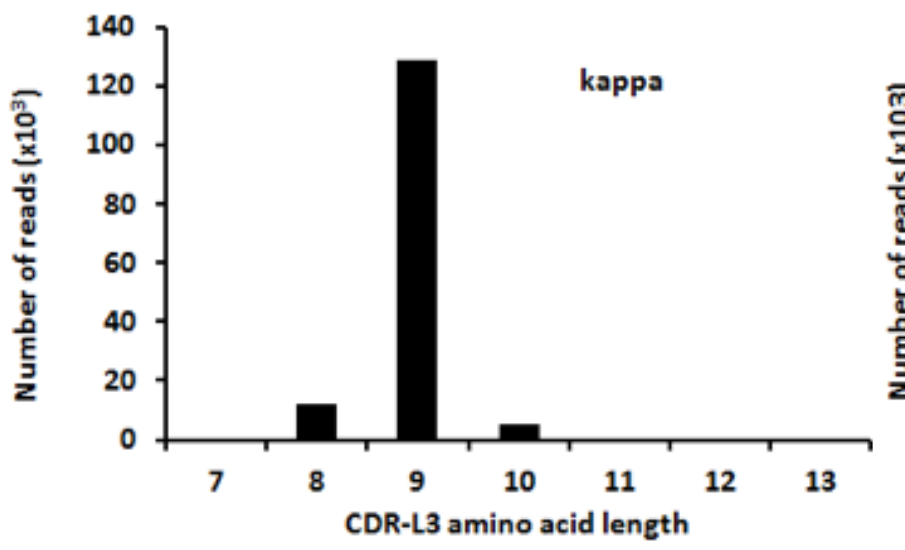

B

432

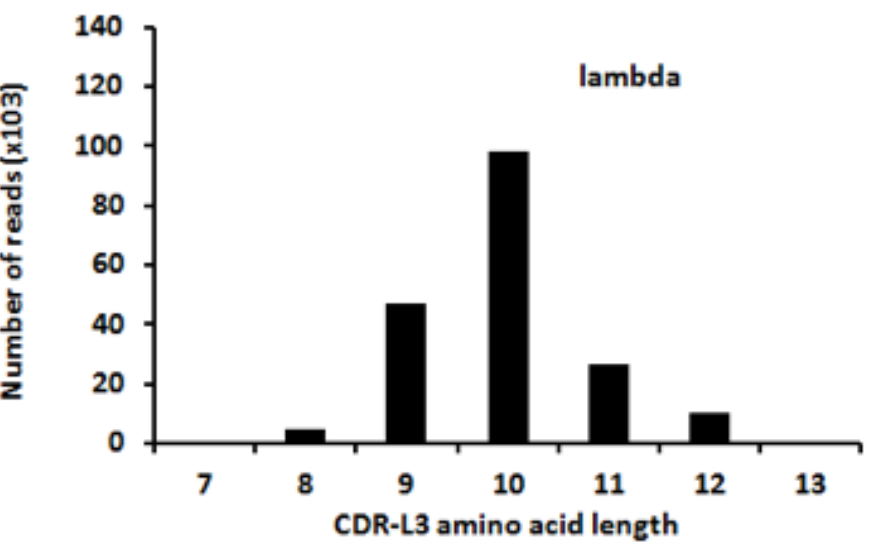


433 Figure 3. Light chain abundance distributions in PRRSV-infected (open circles) and uninfected (closed

434 circles) pigs. (A) Relative abundance of lambda sequences as a proportion of the total lambda repertoire.

435 (B) Effect of PRRSV infection on the abundance of unique lambda clones (data on Y-axis of panel A,

436 shown on a linear scale). (C) Relative abundance of kappa sequences as a proportion of the total kappa

437 repertoire. (D) Effect of PRRSV infection on the abundance of unique kappa clones (data on Y-axis of

438 panel C, shown on a linear scale).

439

A

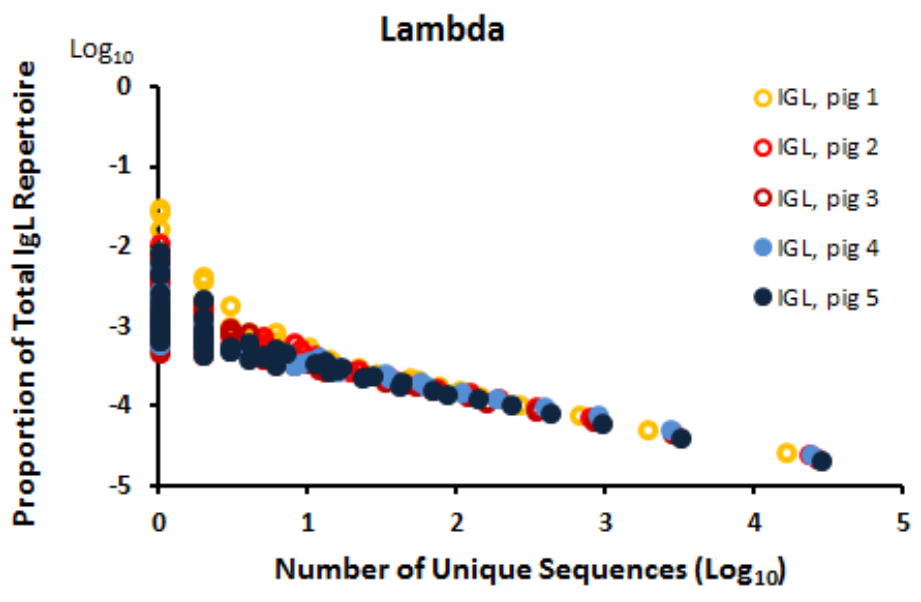

C

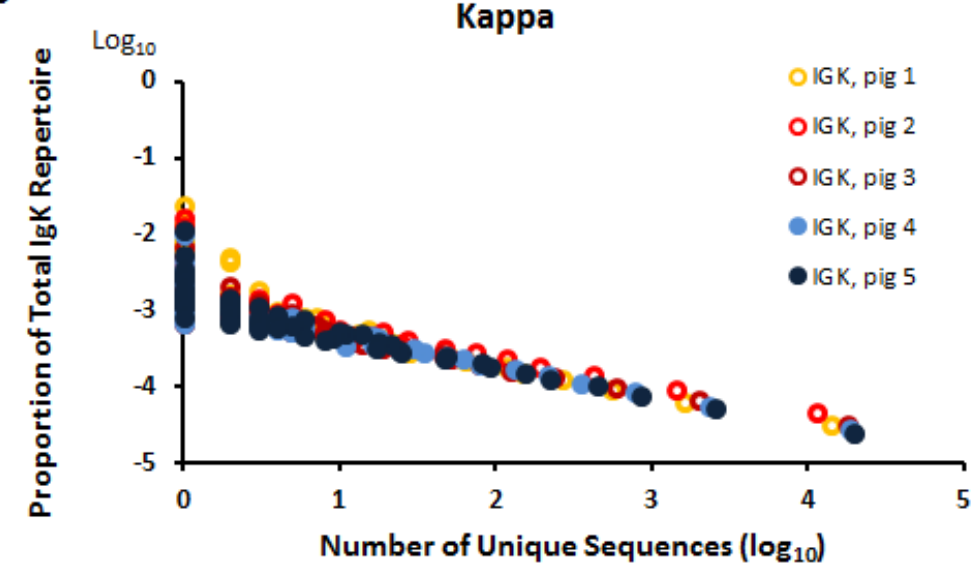

B



D

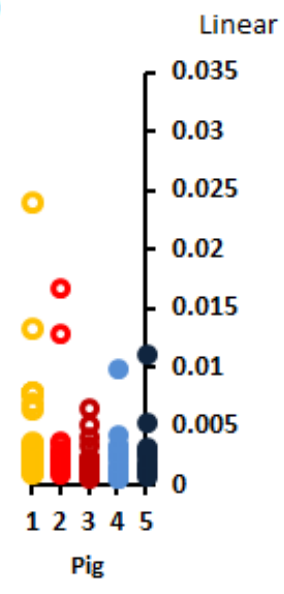


443 Figure 4. Lower-bound Chao1 richness estimates for the total size of the light chain repertoire among

444 individual pigs. Lambda: average, $2.3 \times 10^{5}$; standard deviation: $\pm 4.9 \times 10^{4}$. Kappa: average, $1.5 \times 10^{5}$;

445 standard deviation, $\pm 4.9 \times 10^{4}$.

446

$\angle$

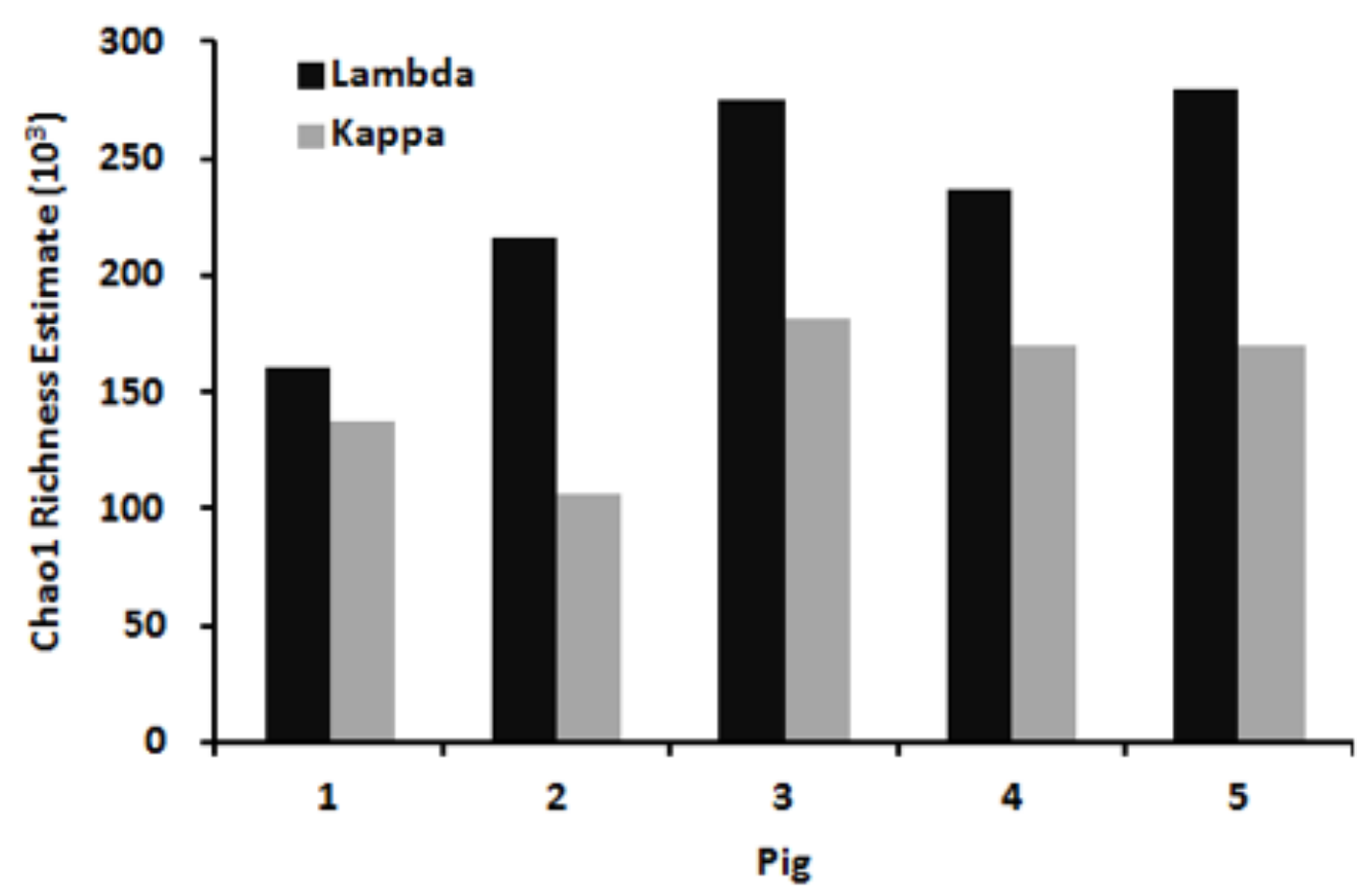


bioRxiv preprint doi: https://doi.org/10.1101/398529; this version posted August 23, 2018. The copyright holder for this preprint (which was not certified by peer review) is the author/funder, who has granted bioRxiv a license to display the preprint in perpetuity. It is made available under aCC-BY-NC-ND 4.0 International license.

448 Figure 5. Distribution of amino acid sequence variation across light chain VJ region. The

449 distribution for IGLV3 includes IGLV3-3, IGLV3-4, IGLV3-5, and IGLV3-6 for which the former

450 is also shown separate due its greater genetic distance from the other three genes.

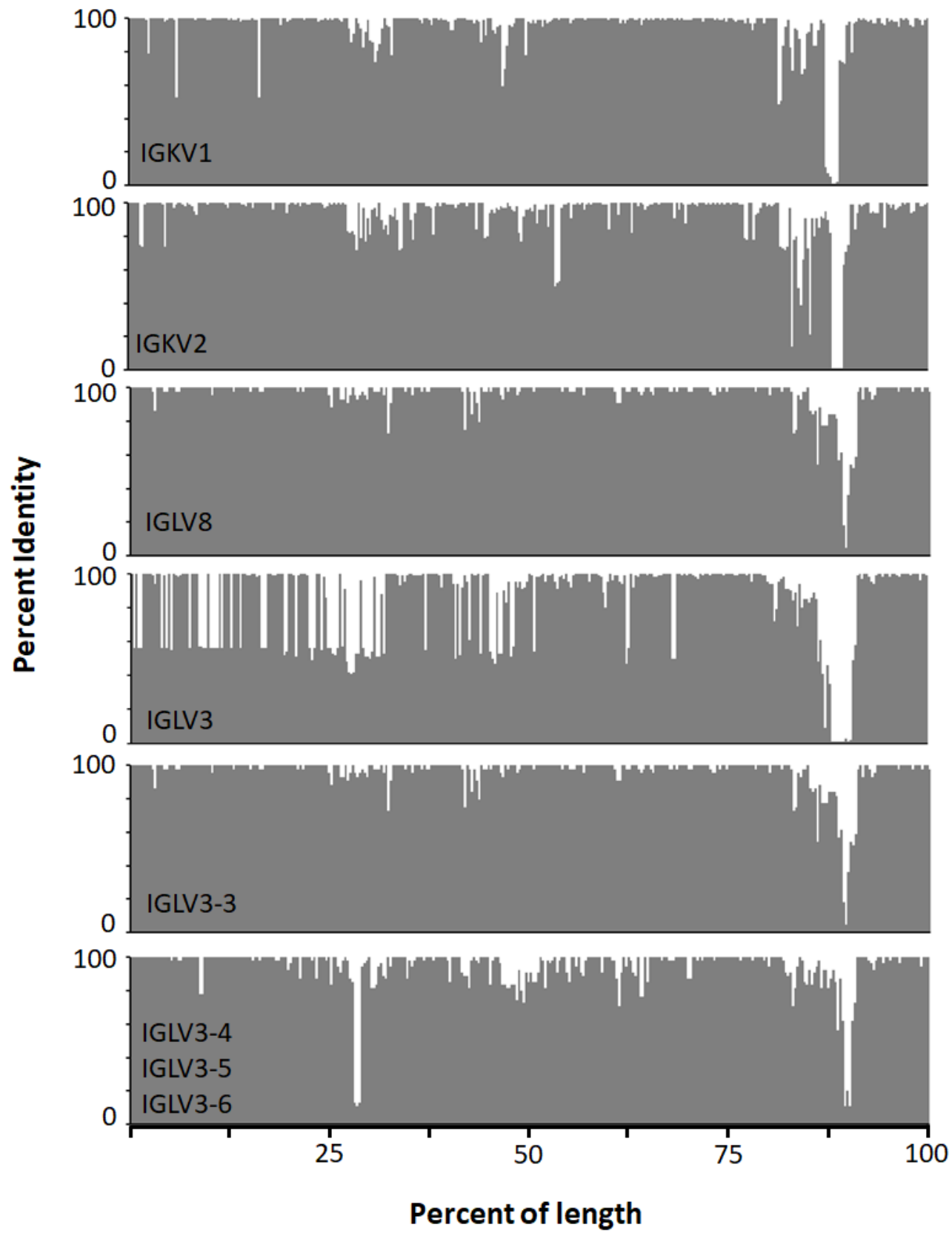

